PROCEEDINGS OF THE

AMERICAN MATHEMATICAL SOCIETY

Volume 127, Number 5, Pages 1357-1362

S 0002-9939(99)04830-3

Article electronically published on January 28, 1999

\title{
CONTROLLING AND SUPPORTING SETS IN OPERATOR ALGEBRAS
}

\author{
CHARLES BURNAP AND ALAN LAMBERT
}

(Communicated by David R. Larson)

\begin{abstract}
Two properties of subspaces of Banach algebras are introduced. These are closely related to Mathieu's concept of ultraprime Banach algebras. Special attention is paid to nested $C^{*}$ and $W^{*}$ algebras.
\end{abstract}

\section{INTRODUCTION}

Let $\mathcal{A}$ be a Banach algebra. Then, for each $a, b \in \mathcal{A}$ the bounded linear operator $\mathbf{M}_{a, b}: \mathcal{A} \rightarrow \mathcal{A}$ is defined by $M_{a, b} x=a x b$. Linear combinations of such operators are called elementary operators, and have been widely studied in recent years (see, for example, the collection of articles in [M3]). As part of his development of the study of elementary operators and related topics, M. Mathieu introduced (in [M1]) the idea of an ultraprime Banach algebra. An algebra $\mathcal{A}$ is defined to be ultraprime if there is a positive number $c$ such that $c\|a\|\|b\| \leq\left\|M_{a, b}\right\|$ for all $a, b \in \mathcal{A}$. In this note we shall introduce and examine two concepts closely related to this idea. Indeed, both new concepts have to do with subsets of a given Banach space forcing a type of local ultraprime behavior on $\mathcal{A}$. As an application of these ideas, we give a proof of a conjecture of J. Deddens. Although this conjecture has been confirmed independently by (at least) three authors, the techniques employed here should be applicable in other Banach algebra investigations.

Definition. Let $\mathcal{A}$ be a Banach algebra and let $\mathcal{K}$ be a nonempty subset of $\mathcal{A}$ other than $\{0\}$. For $a$ and $b$ in $\mathcal{A}$, let $\left\|M_{a, b} \mid \mathcal{K}\right\|=\sup \left\{\frac{\|a k b\|}{\|k\|}: k \in \mathcal{K},\|k\| \neq 0\right\}$. We say that $\mathcal{K}$ is controlling in $\mathcal{A}$ if there is a positive number $e$ such that

$$
e\|a\|\|b\| \leq\left\|M_{a, b} \mid \mathcal{K}\right\| \quad \text { for all } a, b \in \mathcal{A} .
$$

We say that $\mathcal{K}$ is supporting in $\mathcal{A}$ if there is a positive number $d$ such that

$$
d\left\|M_{a, b}\right\| \leq\left\|M_{a, b} \mid \mathcal{K}\right\| \quad \text { for all } a, b \in \mathcal{A} .
$$

Note that $\mathcal{A}$ is ultraprime if and only if $\mathcal{A}$ is controlling in $\mathcal{A}$.

Notation. The collection of sets which are controlling in $\mathcal{A}$ is denoted by $c(\mathcal{A})$. Similarly, $s(\mathcal{A})$ denotes the collection of sets which are supporting in $\mathcal{A}$.

Received by the editors August 6, 1997.

1991 Mathematics Subject Classification. Primary 46L05, 46H20, 47 B47.

(c)1999 American Mathematical Society 
The proof of the following proposition is straightforward and omitted.

1. Proposition. Let $\mathcal{A}$ be a Banach algebra and let $\mathcal{K}$ be a nonempty, nonzero subset of $\mathcal{A}$. Then

(a) $\mathcal{A}$ is ultraprime if and only if for all sequences $\left\{a_{n}\right\},\left\{b_{n}\right\}$ in $\mathcal{A}, \sup _{n}\left\|M_{a_{n}, b_{n}}\right\|$ $<\infty$ implies $\sup _{n}\left\|a_{n}\right\|\left\|b_{n}\right\|<\infty$,

(b) $\mathcal{K}$ is controlling in $\mathcal{A}$ if and only if for all sequences $\left\{a_{n}\right\},\left\{b_{n}\right\}$ in $\mathcal{A}$,

$$
\sup _{n}\left\|M_{a_{n}, b_{n}} \mid \mathcal{K}\right\|<\infty \quad \text { implies } \sup _{n}\left\|a_{n}\right\|\left\|b_{n}\right\|<\infty,
$$

(c) $\mathcal{K}$ is supporting in $\mathcal{A}$ if and only if for all sequences $\left\{a_{n}\right\},\left\{b_{n}\right\}$ in $\mathcal{A}$,

$$
\sup _{n}\left\|M_{a_{n}, b_{n}} \mid \mathcal{K}\right\|<\infty \quad \text { implies } \sup _{n}\left\|M_{a_{n}, b_{n}}\right\|<\infty .
$$

The next result establishes some relationships among the three properties described above. The proof is followed by an example showing that the set inclusion in part (a) may be proper. Of course this can also be deduced from part (b), but it seems appropriate at this point to provide an explicit example.

2. Proposition. Let $\mathcal{A}$ be a Banach algebra. Then

(a) $c(\mathcal{A}) \subset s(\mathcal{A})$.

(b) $c(\mathcal{A})=s(\mathcal{A})$ if and only if $\mathcal{A}$ is ultraprime.

Proof. (a) Suppose $\mathcal{K} \in c(\mathcal{A})$; then with $e>0$ as in the definition of controlling, we have for all $a$ and $b$ in $\mathcal{A}$,

$$
e\left\|M_{a, b}\right\| \leq e\|a\|\|b\| \leq\left\|M_{a, b} \mid \mathcal{K}\right\|,
$$

hence $\mathcal{K} \in s(\mathcal{A})$.

(b) Clearly, $\mathcal{A}$ is always supporting in $\mathcal{A}$, but if $\mathcal{A}$ is a Banach algebra that is not ultraprime, then $\mathcal{A} \notin c(\mathcal{A})$, hence $c(\mathcal{A}) \neq s(\mathcal{A})$.

Conversely, suppose $\mathcal{A}$ is ultraprime. If $\mathcal{K} \in s(\mathcal{A})$, then there are constants $c, d>0$ such that for all $a, b \in \mathcal{A}$,

$$
c d\|a\|\|b\| \leq d\left\|M_{a, b}\right\| \leq\left\|M_{a, b} \mid \mathcal{K}\right\| .
$$

This shows $\mathcal{K} \in c(\mathcal{A})$, hence $s(\mathcal{A}) \subset c(\mathcal{A})$. In view of part (a), we have $c(\mathcal{A})=$ $s(\mathcal{A})$.

The ultraprime concept is not, in general, of much use when $\mathcal{A}$ is abelian, since in that case $\mathbf{M}_{a, b}$ is the operator of multiplication by $a b$, and there is in general no hope of bounding $\|a\| \cdot\|b\|$ above by a scalar multiple of $\left\|M_{a, b}\right\|$. This lack of applicability carries over to controlling sets as well. However the idea of a supporting set may still be useful in the abelian setting. Consider the case of $\mathcal{A}=l^{\infty}$ and $\mathcal{K}=c_{0}$. For $a=$ $\left(a_{i}\right)$ and $b=\left(b_{i}\right)$ in $\mathcal{A},\left\|M_{a, b}\right\|=\|a \cdot b\|_{\infty}$. Let $x_{n}=(1,1, \ldots, 1,0,0, \ldots) \in c_{0}$ (the last 1 in position $n$ ). Then $\left\|x_{n}\right\|=1$ for all $n$, and $\left\|M_{a, b} x_{n}\right\|=\max _{1 \leq i \leq n}\left|a_{i} b_{i}\right| \rightarrow$ $\|a \cdot b\|_{\infty}$. Thus $\left\|M_{a, b}\right\|=\left\|M_{a, b} \mid c_{0}\right\|$, and so $c_{0} \in s\left(l^{\infty}\right)$. By choosing $a$ and $b$ to be unit vectors in $l^{\infty}$ with disjoint supports, we see that $\left\|M_{a, b} \mid c_{0}\right\|=\left\|M_{a, b}\right\|=0$, so that $c_{0}$ is not controlling in $l^{\infty}$.

\section{Support AND CONTROL IN $C^{*}$-ALGEBRAS}

In what follows, for $\mathcal{S}$ a subset of an algebra $\mathcal{A}, \mathcal{S}^{\prime}$ denotes the commutant of $\mathcal{S}$; that is, $\mathcal{S}^{\prime}=\{a \in \mathcal{A}: \forall s \in \mathcal{S}$, as $=s a\}$. 
3. Theorem. (a) Let $\mathcal{B}$ be a $C^{*}$ subalgebra of a von Neumann algebra $\mathcal{A}$. If $\mathcal{B}$ is weakly dense in $\mathcal{A}$, then $\mathcal{B} \in s(\mathcal{A})$.

(b) Let $\mathcal{H}$ be a Hilbert space and let $\mathcal{B}$ be a $C^{*}$-subalgebra of $\mathcal{L}(\mathcal{H})$, the ring of all bounded operators on $\mathcal{H}$. Then $\mathcal{B}$ is weakly dense in $\mathcal{L}(\mathcal{H})$ if and only if $\mathcal{B} \in s(\mathcal{L}(\mathcal{H}))$.

Proof. (a) Suppose $\mathcal{B}$ is weakly dense in $\mathcal{A}$. Then, given $T \in \mathcal{A}$, there is a net $\left\{B_{\lambda}\right\}$ in $\mathcal{B}$ such that $\left\{B_{\lambda}\right\}$ converges weakly to $T$. Using the Kaplansky Density Theorem, we may assume that $\left\|B_{\lambda}\right\| \leq\|T\|$ for all $\lambda$. For $A, C \in \mathcal{A}$, we have $A B_{\lambda} C \stackrel{w}{\longrightarrow} A T C$. Furthermore, since the unit ball in $\mathcal{A}$ is weakly compact, we see that

$$
\begin{aligned}
& \|A T C\| \leq \limsup _{\lambda}\left\|A B_{\lambda} C\right\| \leq\left\|M_{A, C} \mid \mathcal{B}\right\| \limsup _{\lambda}\left\|B_{\lambda}\right\| \\
& \leq\left\|M_{A, C} \mid \mathcal{B}\right\|\|T\| .
\end{aligned}
$$

Taking the supremum over $T$ in the unit ball of $\mathcal{A}$, we conclude that $\left\|M_{A, C}\right\|=$ $\left\|M_{A, C} \mid \mathcal{B}\right\|$, hence $\mathcal{B} \in s(\mathcal{A})$.

(b) By part (a), we need only show that if $\mathcal{B} \in s(\mathcal{L}(\mathcal{H}))$, then $\mathcal{B}$ is weakly dense in $\mathcal{L}(\mathcal{H})$. Suppose $\mathcal{B}$ is not weakly dense in $\mathcal{L}(\mathcal{H})$. Then there is an orthogonal projection $P \in \mathcal{B}^{\prime}$ with $P \neq 0, P \neq I$. For each $n \in \mathbb{N}$, set $A_{n}=n P+\frac{1}{n}(I-P)$ and $C_{n}=A_{n}^{-1}=\frac{1}{n} P+n(I-P)$. (As usual $I$ denotes the identity operator.) Now $A_{n}, C_{n} \in \mathcal{B}^{\prime}$ implies that $M_{A_{n}, C_{n}} \mid \mathcal{B}$ is the identity operator on $\mathcal{B}$ for every $n \in \mathbb{N}$. Thus, $\left\|M_{A_{n}, C_{n}} \mid \mathcal{B}\right\|=1$. On the other hand, if we let $u, v$ be unit vectors in $\mathcal{H}$ such that $P u=0$ and $P v=v$ and define $u \otimes v: \mathcal{H} \rightarrow \mathcal{H}$ by $(u \otimes v) w=\langle w, u\rangle v$, we have

$$
\begin{aligned}
A_{n}(u \otimes v) C_{n} & =\left(C_{n}^{*} u\right) \otimes\left(A_{n} v\right) \\
& =\left(C_{n} u\right) \otimes\left(A_{n} v\right) \quad\left(C_{n} \text { is selfadjoint }\right) \\
& =(n u) \otimes(n v) .
\end{aligned}
$$

Therefore $\left\|M_{A_{n}, C_{n}}\right\| \geq n^{2}$ for all $n \in \mathbb{N}$, and consequently $\mathcal{B} \notin s(\mathcal{L}(\mathcal{H}))$.

Remarks. 1. Mathieu has established the elegant fact that a von Neumann algebra is ultraprime if and only if it is a factor $([\mathrm{M} 2])$. In particular $\mathcal{L}(\mathcal{H})$ is ultraprime and part (b) of Proposition 2 yields $s(\mathcal{L}(\mathcal{H}))=c(\mathcal{L}(\mathcal{H}))$. Thus the conclusion of Theorem 3 is equivalent to " $\mathcal{B}$ is weakly dense in $\mathcal{L}(\mathcal{H})$ if and only if $\mathcal{B} \in c(\mathcal{L}(\mathcal{H}))$." We shall make use of this fact later in this note.

2. The example following Proposition 2 gives a nonfactor von Neumann algebra $\left(l^{\infty}\right.$ acting on $\left.l^{2}\right)$ and a weakly dense ${ }^{*}$-subalgebra $\left(c_{0}\right)$ which is supporting but not controlling.

3. The second part of the preceding proof is applicable in more general circumstances. For example, suppose that the nonabelian algebra $\mathcal{A}$ has unit 1 and $P$ is an idempotent in $\mathcal{A}$ other than 0 or 1 . Suppose also that $K$ is a member of $\mathcal{A}$ for which $P K(1-P) \neq 0$. Then no subset of $\{P\}^{\prime}$ can be supporting for $\mathcal{A}$. Indeed, if $\mathcal{B} \subset\{P\}^{\prime}$ and $A_{n}$ and $C_{n}$ are defined in terms of $P$ as in the proof of Theorem 3, then $\left\|M_{A_{n}, C_{n}} K\right\|=\left\|P K P+(1-P) K(1-P)+n^{2} P K(1-P)+\frac{1}{n^{2}}(1-P) K P\right\|$. Thus $\lim _{n \rightarrow \infty}\left\|M_{A_{n}, C_{n}} K\right\|=\infty$, while for all $n,\left\|M_{A_{n}, C_{n}} \mid \mathcal{B}\right\|=1$.

4. The proof presented for part (b) of Theorem 3 works in the more general setting of part (a) so long as the relative commutant $\mathcal{B}^{\prime} \cap \mathcal{A}$ is nontrivial. It is shown below (Proposition 7 ) that if this relative commutant is nontrivial, then $\mathcal{B}$ cannot be controlling for $\mathcal{A}$. We do not know if the converse of part (a) holds in general. 
Our solution to the problem posed by Deddens alluded to in the introduction of this article is based on the following corollaries. (A precise statement of Deddens' problem is contained in the remarks below.)

4. Corollary. Let $\mathcal{H}$ be a Hilbert space and let $\mathcal{K}(\mathcal{H})$ denote the collection of compact operators on $\mathcal{H}$. Then

$$
\mathcal{K}(\mathcal{H}) \in c(\mathcal{L}(\mathcal{H}))=s(\mathcal{L}(\mathcal{H})) .
$$

Proof. $\mathcal{K}(\mathcal{H})$ is a weakly dense $C^{*}$ subalgebra of $\mathcal{L}(\mathcal{H})$.

5. Corollary. Let $\left\{A_{n}\right\}$ and $\left\{B_{n}\right\}$ be sequences of operators on a Hilbert space $\mathcal{H}$ such that for all compact $K, \sup _{n}\left\|A_{n} K B_{n}\right\|<\infty$. Then $\sup _{n}\left\|A_{n}\right\|\left\|B_{n}\right\|<\infty$.

Proof. For each $n \in \mathbb{N}, M_{A_{n}, B_{n}}$ is pointwise bounded on $\mathcal{K}(\mathcal{H})$ hence, by the uniform boundedness principle, $\left\|M_{A_{n}, B_{n}} \mid \mathcal{K}(\mathcal{H})\right\|$ is bounded. Since $\mathcal{K}(\mathcal{H})$ is controlling in $\mathcal{L}(\mathcal{H})$, the conclusion follows.

Remarks. 1. Although Corollaries 4 and 5 were stated in * algebra format, the results remain valid when $K(\mathcal{H})$ is replaced by $\mathcal{O}$, the set of rank one operators on $\mathcal{H}$; i.e., $\mathcal{O} \in c(\mathcal{L}(\mathcal{H}))$. Indeed, for $A$ and $B$ in $\mathcal{L}(\mathcal{H})$ and unit vectors $u$ and $v$ in $\mathcal{H}$,

$$
\|A(u \otimes v) B\|=\left\|B^{*} u \otimes A v\right\|=\left\|B^{*} u\right\|\|A v\| .
$$

This insures that $\left\|M_{A, B} \mid \mathcal{O}\right\|=\|A\|\|B\|$.

2. Suppose $A$ is an invertible operator. Then, applying the preceding remark to the sequences $\left\{A^{n}\right\},\left\{A^{-n}\right\}$, we see that if $\mathcal{O} \subset\left\{K \in \mathcal{L}(\mathcal{H}): \sup _{n}\left\|A^{n} K A^{-n}\right\|<\right.$ $\infty\}$, then $\sup _{n}\left\|A^{n}\right\|\left\|A^{-n}\right\|<\infty$. This finiteness condition is in fact necessary and sufficient to deduce that $A$ is similar to a multiple of a unitary operator ([Sz-N]). This relates directly to the following problem raised originally by J. Deddens in [D]:

Given an invertible operator $A$ for which the sequence $\left\|A^{n} K A^{-n}\right\|$ is bounded for every $K \in \mathcal{L}(\mathcal{H})$, must $A$ be similar to a scalar multiple of a unitary operator?

Affirmative answers were provided independently by J. Stampfli ([S]), D. Herrero ([H1], [H2]), and J. P. Williams ([W]). These authors all noted that, in fact, it suffices to hypothesize only the boundedness of $\left\|A^{n} K A^{-n}\right\|$ for all rank one operators $K$.

We now examine some questions about controlling von Neumann algebras.

6. Proposition. Suppose $\mathcal{V}$ and $\mathcal{W}$ are von Neumann subalgebras of $\mathcal{L}(\mathcal{H})$ for some Hilbert space $\mathcal{H}$. If $\mathcal{W}$ is a type I factor and $\mathcal{V}$ is a controlling subspace in $\mathcal{W}$, then $\mathcal{V}=\mathcal{W}$.

Proof. Given a type I factor $\mathcal{W}$ there is a Hilbert space $\mathcal{J}$ and an isometric isomorphism $\phi$ mapping $\mathcal{W}$ onto $\mathcal{L}(\mathcal{J})([\mathrm{T}])$. Now $\mathcal{V} \in c(\mathcal{W})$ if and only if there is an $e>0$ such that for all $w_{1}, w_{2} \in \mathcal{W}$

$$
e\left\|w_{1}\right\|\left\|w_{2}\right\| \leq\left\|M_{w_{1}, w_{2}} \mid \mathcal{V}\right\| .
$$

Because $\phi$ is isometric, the inequality above is equivalent to

$$
e\left\|\phi\left(w_{1}\right)\right\|\left\|\phi\left(w_{2}\right)\right\| \leq\left\|\phi \circ\left(M_{w_{1}, w_{2}} \mid \mathcal{V}\right)\right\| .
$$

When $w \in \mathcal{V}$ we have

$$
M_{\phi\left(w_{1}\right), \phi\left(w_{2}\right)} \phi(w)=\phi\left(M_{w_{1}, w_{2}} w\right)
$$


hence

$$
M_{\phi\left(w_{1}\right), \phi\left(w_{2}\right)} \mid \phi(\mathcal{V})=\phi \circ\left(M_{w_{1}, w_{2}} \mid \mathcal{V}\right)
$$

and we may rewrite (2) as

$$
e\left\|\phi\left(w_{1}\right)\right\|\left\|\phi\left(w_{2}\right)\right\| \leq\left\|M_{\phi\left(w_{1}\right), \phi\left(w_{2}\right)} \mid \phi(\mathcal{V})\right\| .
$$

Because $\phi$ maps $\mathcal{W}$ onto $\mathcal{L}(\mathcal{J})$ and (1) is equivalent to (3), we conclude that $\mathcal{V} \in$ $c(\mathcal{W})$ if and only if $\phi(\mathcal{V}) \in c(\mathcal{L}(\mathcal{J}))$. Utilizing the remark following Theorem 3, we see that the statement $\phi(\mathcal{V}) \in c(\mathcal{L}(\mathcal{J}))$ is equivalent to $\phi(\mathcal{V})=\mathcal{L}(\mathcal{J})$, hence to $\mathcal{V}=\mathcal{W}$.

7. Proposition. Suppose $\mathcal{V}$ and $\mathcal{W}$ are von Neumann algebras such that $\mathcal{V} \subset \mathcal{W}$ and $\mathcal{V} \in c(\mathcal{W})$. Then $\mathcal{V}^{\prime} \cap \mathcal{W}=\mathbb{C} I$.

Proof. Assume, to the contrary, that $\mathcal{V}^{\prime} \cap \mathcal{W} \neq \mathbb{C} I$ and let $P$ be an orthogonal projection in $\mathcal{V}^{\prime} \cap \mathcal{W}$ other than 0 or $I$. As in the proof of Theorem 3, for each $n \in \mathbb{N}$, we set $A_{n}=n P+\frac{1}{n}(I-P)$ and $C_{n}=A_{n}^{-1}=\frac{1}{n} P+n(I-P)$. Now $P \in \mathcal{V}^{\prime}$ so $\left\|M_{A_{n}, C_{n}} \mid \mathcal{V}\right\|=1$, but, for each $n \in \mathbb{N}$, $\left\|A_{n}\right\|\left\|A_{n}^{-1}\right\|=n^{2}$. This contradicts the supposition that $\mathcal{V} \in c(\mathcal{W})$ and the desired conclusion follows.

Proposition 7 gives another proof of Mathieu's result that the only ultraprime von Neumann algebras are factors (recall that $\mathcal{A}$ is controlling in $\mathcal{A}$ if and only if $\mathcal{A}$ is ultraprime):

8. Corollary. If $\mathcal{V}$ is controlling in $\mathcal{V}$, then $\mathcal{V}$ is a factor, i.e. $\mathcal{V}^{\prime} \cap \mathcal{V}=\mathbb{C} I$.

Note that we could not use the proof given in Theorem 3 to prove that $\mathcal{V} \in s(\mathcal{W})$ implies $\mathcal{V}^{\prime} \cap \mathcal{W}=\mathbb{C} I$ because the rank one operators $u \otimes v$ need not be in $\mathcal{W}$. (If they were, then since $\mathcal{V}$ is always supporting in $\mathcal{V}$, we must have $\mathcal{V}^{\prime} \cap \mathcal{V}=\mathbb{C} I$.)

9. Corollary. If $\mathcal{V}$ and $\mathcal{W}$ are von Neumann algebras with $\mathcal{V} \subset \mathcal{W}$ and $\mathcal{V} \in c(\mathcal{W})$, then both $\mathcal{V}$ and $\mathcal{W}$ are factors.

Proof. Note that $\mathcal{V}^{\prime} \cap \mathcal{W}$ contains both centers.

Question. Are there non-type I factors with proper controlling subfactors?

We end this note with the establishment of a method for obtaining controlling sets from other controlling sets.

10. Theorem. Suppose $\mathcal{B}$ is controlling in a Banach algebra $\mathcal{A}, a \in \mathcal{A}$, and suppose that there is a $\delta>0$ such that for all $x \in \mathcal{A},\left\|a^{2} x\right\| \geq \delta \|$ ax $\|$ and $\left\|x a^{2}\right\| \geq \delta\|x a\|$. Then $[a \mathcal{B} a]$ is controlling in $[a \mathcal{A} a]$. (The brackets indicate closed linear span.)

Proof. Let $a_{1}, a_{2} \in \mathcal{A}$ and let $b \in \mathcal{B}$. Then

$$
\left\|\left(a a_{1} a\right)(a b a)\left(a a_{2} a\right)\right\|=\left\|\left(a a_{1} a^{2}\right) b\left(a^{2} a_{2} a\right)\right\|
$$

so that

$$
\sup _{b \in \mathcal{B}_{1}}\left\|\left(a a_{1} a\right)(a b a)\left(a a_{2} a\right)\right\| \geq\left\|M_{a a_{1} a^{2}, a^{2} a_{2} a} \mid \mathcal{B}\right\| \geq d\left\|a a_{1} a^{2}\right\|\left\|a^{2} a_{2} a\right\|
$$

where $\mathcal{B}_{1}$ is the unit ball in $\mathcal{B}$. The existence of the constant $d>0$ is assured because $\mathcal{B}$ is controlling in $\mathcal{A}$. Now, using the additional inequalities given in the hypothesis of the theorem, we obtain

$$
\sup _{b \in \mathcal{B}_{1}}\left\|\left(a a_{1} a\right)(a b a)\left(a a_{2} a\right)\right\| \geq d \delta^{2}\left\|a a_{1} a\right\|\left\|a a_{2} a\right\| .
$$


Thus,

$$
\begin{aligned}
\left\|M_{a a_{1} a, a a_{2} a} \mid a \mathcal{B} a\right\|= & \sup \left\{\left\|\left(a a_{1} a\right)(a y a)\left(a a_{2} a\right)\right\|: y \in \mathcal{B},\|a y a\| \leq 1\right\} \\
\geq & \sup \left\{\left\|\left(a a_{1} a\right) \frac{(a b a)}{\left\|a^{2}\right\|}\left(a a_{2} a\right)\right\|: b \in \mathcal{B}_{1}\right\} \\
& \left(\operatorname{set} b=\left\|a^{2}\right\| y\right) \\
\geq & \frac{d \delta^{2}}{\left\|a^{2}\right\|}\left\|a a_{1} a\right\|\left\|a a_{2} a\right\| \quad \text { (using (4)) }
\end{aligned}
$$

and, therefore, $[a \mathcal{B} a]$ is controlling in $[a \mathcal{A} a]$.

11. Corollary. Suppose $a$ is an element of an ultraprime Banach algebra $\mathcal{A}$. If there is $a \delta>0$ such that for all $x \in \mathcal{A},\left\|a^{2} x\right\| \geq \delta\|a x\|$ and $\left\|x a^{2}\right\| \geq \delta\|x a\|$, then $[a \mathcal{A} a]$ is ultraprime.

12. Corollary. Suppose $\mathcal{W}$ is an ultraprime von Neumann algebra (i.e. a factor) and $T=T^{*} \in \mathcal{W}$. If there is a $\delta>0$ such that for all $w \in \mathcal{W},\left\|T^{2} w\right\| \geq \delta\|T w\|$, then $[T \mathcal{W} T]$ is ultraprime.

\section{REFERENCES}

[D] J. A. Deddens, Another Description of Nests, Hilbert Space Operators, Proceedings, Long Beach, California, 1977. Lect. Notes Math. 693, Springer-Verlag, New York, 1978, 77-86. MR 80f: 47033

[H1] D. A. Herrero, On iterated similarities of operators, Proc. Amer. Math. Soc. 72 (1978), 519-520. MR 80e:47014

[H2] D. A. Herrero, A note on iterated similarities of operators, Revista de la Unión Matemática Argentina 36 (1990), 138-145. MR 94k:47029

[M1] M. Mathieu, Applications of ultraprime Banach algebras in the theory of elementary operators, Ph.D. dissertation, Universität Tübingen, 1986.

[M2] Spectral theory for multiplication operators on $C^{*}$-algebras, Proc. Royal Irish Acad. 83A (1983), 231-249. MR 85m:46055

[M3] _ Ed., Elementary Operators and Applications, World Scientific, River Edge, N.J., 1992. MR 93e: 47001

[S] J. G. Stampfli, On a Question of Deddens, Hilbert Space Operators, Proceedings, Long Beach, California, 1977. Lecture Notes Math. 693, Springer-Verlag, New York, 1978, 169173. MR 80f: 47034

[Sz-N] B. Sz-Nagy, On uniformly bounded linear transformations in Hilbert space, Acta Scient. Math. (Szeged), 11 (1947), 152-157.

[T] D. M. Topping, Lectures on von Neumann Algebras, Van Nostrand Reinhold Co., London 1971.

[W] J. P. Williams, On a boundedness condition for operators with a singleton spectrum, Proc. Amer. Math. Soc. 78 (1980), 30-32. MR 81k:47008

Department of Mathematics, The University of North Carolina at Charlotte, CharLOTTE, NorTh CAROlina 28223

E-mail address: caburnap@email.uncc.edu

E-mail address: allamber@email.uncc.edu 